Pacific Journal of Mathematic 


\title{
EVERY SPACE IS A PATH COMPONENT SPACE
}

\section{DOUGLas HARRIS}

\begin{abstract}
It is shown that every topological space appears as quotient of a paracompact Hausdorff space in a very natural way: as the space of path components. This means that the "pathology" of closure operators in non-Hausdorff spaces appears as "pathology" of path components in spaces which have "nice" closure operators.
\end{abstract}

1. Introduction. There has not been much general interest in non-Hausdorff spaces since their behavior seems in many respects pathological. Even general Hausdorff spaces still exhibit certain bizarre behavior. As a result the majority of topologists have concentrated their attention on completely regular Hausdorff spaces, usually including an additional property such as compactness or paracompactness, to insure desirable characteristics such as normality.

There is one type of behavior among such spaces that is sometimes thought of as pathological, but it is well understood and has not led general topologists to disallow it via axioms. This is the behavior of path components. They need not be closed sets, and the closure of a path component may have an arbitrarily complex structure.

In this paper it will be shown that the two types of pathology are closely related; in particular that any topological space expresses abstractly the structure of the path components in an appropriately constructed paracompact Hausdorff space. Specifically, a functor $S$ is constructed from the category of topological spaces and maps to the category of paracompact Hausdorff spaces such that the original space $X$ is the path component space of the image $S(X)$. Furthermore it is shown that the functor may be extended to an embedding into the category of compact Hausdorff spaces, although recovery is no longer done simply through the path component space functor.

The constructions of this paper also give a representation of any topological space as quotient of a paracompact Hausdorff space. To find such a representation is not difficult, but the particular representation given has the rather unusual property that the components in the representing space are in one-one correspondence with the components in the represented space; in particular a connected space is the quotient of a connected space. Also the decomposition of the representing space need not be specified in advance, but can be naturally recovered as the path component decomposition. 
The results of this paper do not show that one need not deal with arbitrary spaces directly. The passage from $X$ to $S(X)$ is artificial and generally cannot be used to deduce new properties of the space $X$. Rather the results show that the category of paracompact Hausdorff spaces is itself very complex and contains hidden within all the complexities of Top. The passage from $X$ to $S(X)$ allows one to begin with a simple but strange space $X$ and to construct a more complex space $S(X)$ which has many convenient properties and in which the strangeness of $X$ becomes more familiar.

In the remainder of this paper the term space means an arbitrary topological space and the term map means a continuous function between spaces.

2. Construction of $S(X)$. Let $R$ represent the positive reals with their usual topology. A subset $A$ of $R$ is coinitial if it is not bounded away from 0 , initial if it contains an entire interval from 0 , cofinal if it contains infinitely many integers, and terminal if it contains an entire tail of integers. Initial and terminal sets will be used to define the topology of $S(X)$.

For each pair $x, y$ of points in $X$ let $R_{x}^{y}$ be a copy of $R$, and let $S_{x}$ be the set-theoretic disjoint union of a singleton $\left\{x_{0}\right\}$ with the set $\left\{R_{x}^{y}: y \in X\right\}$. The ground set of the space $S(X)$ will be the disjoint union of the collection $\left\{S_{x}: x \in X\right\}$, and $\pi: S(X) \rightarrow X$ will be the natural projection that takes $S_{x}$ to the point $x$. The center of $S(X)$ is the set $X_{0}=\left\{x_{0}: x \in X\right\}$ and the remaining are the radial points. Given $A \subset S(X)$ write $A_{0}$ for $A \cap X_{0}$ and $A_{x}^{y}$ for $A \cap R_{x}^{y}$.

A base for neighborhoods of a radial point $p$ is the set of intervals about $p$ on the ray containing $p$. A neighborhood of $x_{0} \in X_{0}$ is $A$ such that $A_{x}^{y}$ is initial for each $y \in X$ and the set $A(x)=$ $\left\{y \in X: A_{y}^{x}\right.$ is terminal $\}$ is a neighborhood in $X$ of $x$. In particular given a neighborhood $V$ of $x$ and positive real numbers $a, b$ there is a uniform neighborhood $V_{x}(a, b)=\left\{x_{0}\right\} \cup\left\{(0, a)_{x}^{y}: y \in X\right\} \cup\left\{(b, \infty)_{y}^{x}: y \in V\right\}$ of $x_{0}$.

A function $f: X \rightarrow Y$ is pseudo-open [1] if when $f \leftarrow(y) \subset$ int $U$ then $y \in \operatorname{int} f[U]$; equivalently, every restriction to a subset of the codomain is a quotient function.

2.1. The function $\pi$ is a pseudo-open mapping of $S(X)$ onto $X$.

Proof. $\pi-[B]=\bigcup_{x \in B} S_{x}=S_{B}$ meets every $R_{x}^{y}$ in an open set, and meets $R_{y}^{x}$ in a terminal set exactly when $y \in B$. It follows that $S_{B}$ is open if and only if $B$ is open. To see that $\pi$ is pseudo-open, suppose $U$ is a neighborhood of $S_{x}$; then $x_{0} \in U$, and $x \in \operatorname{int} U(x) \subset$ 
$\pi[U]$

\section{2. $S(X)$ is a Hausdorff space.}

Proof. Certainly distinct radial points are separated by disjoint neighborhoods. Since $X_{x}(1.1)$ and $X_{y}(1.1)$ are disjoint when $x \neq y$, then distinct central points are separated. If $x_{0} \in X_{0}$ and $p \in R_{y}^{z}$, then choosing $a<p<b$ the neighborhoods $X_{x}(a, b)$ and $(a, b)_{y}^{z}$ of $x_{0}$ and $p$ are disjoint.

\section{3. $S(X)$ is a paracompact space.}

Proof. Let $\alpha$ be an open cover of $S(X)$ and for each $x, y \in X$ set $\alpha_{x}^{y}=\left\{V_{x}^{y}: V \in \alpha\right\}$. Refine each $\alpha_{x}^{y}$ by a locally finite cover $\beta_{x}^{y}$ that has no initial member and no terminal member. For each central point $x_{0}$ choose an open neighborhood $V_{x}$ of $x_{0}$ that lies in some member of $\alpha$ and in $V_{x}(1.1)$. Set $\gamma=\left\{V: V \in \beta_{x}^{y}, \quad x, y \in X\right\} \cup$ $\left\{V_{x}: x_{0} \in X_{0}\right\}$. A central point is in the closure of only one member of $\gamma$ and a radial point is in the closure of only finitely many members of $\gamma$.

The space $S(X)$ actually has a property stronger than paracompactness; it is stratifiable [2], which means that for each open set $U$ there are open sets $\left\{U_{n}\right\}_{k=1}$ such that $U=U U_{n}$, cl $U_{n} \subset U$ for each $n$, and if $U \subset V$ then $U_{n} \subset V_{n}$ for each $n$. Such a space is not only paxacompact, it is perfectly (and thus completely) normal; in particular every closed set is a $G_{\hat{o}}$ and even a zero set.

\section{4. $S(X)$ is a stratifiable space.}

Proof. Let $V$ be an open set; then it is the union of $V \cap X_{0}$ with a collection $\left\{(a, b)_{x}^{y} ; x, y \in X\right\}$ of disjoint nonempty open intervals on rays $R_{x}^{y}$. Let $k(a)$ be the least integer greater than $a$ and let $l(b)$ be the greatest integer less than $b$. Given an interval $(a, b)$, if $a=0$ set $a_{n}=0$ and if $a \neq 0$ set $a_{n}=a+(k(a)-a) / 3 n$; if $b=\infty$ set $b=\infty$ and if $b<\infty$ set $b_{n}=b-(b-1(b)) / 3 n$. Then put $U_{n}=$ $\left(V \cap X_{0}\right) \cup\left\{\left(a_{n}, b_{n}\right)_{x}^{y} \cap(0, n-1 / 3 n)_{x}^{y}: x_{0} \in V, y_{0} \notin V\right\} \cup\left\{\left(a_{n}, b_{n}\right)_{x}^{y} \cap(1 / 3 n, \infty)_{x}^{y}:\right.$ $x_{0} \notin V$ and $\left.y_{0} \in V\right\} \cup\left\{\left(a_{n}, b_{n}\right)_{x}^{y} \cap(1 / 3 n, n-1 / 3 n)_{x}^{y}: x_{0} \notin V\right.$ and $\left.y_{0} \notin V\right\} \cup$ $\left\{\left(a_{n}, b_{n}\right)_{x}^{y}: x_{0} \in V\right.$ and $\left.y_{0} \in V\right\}$.

By construction $V_{n} \subset V$ and $U V_{n}=V$. Clearly radial points in cl $V_{n}$ are in $V_{n+1} \subset V$; if $x_{0} \notin V$ then from the construction $X_{x}(1 / 3 n, n)$ is disjoint from $V_{n}$; thus central points in cl $V_{n}$ are also in $V$. To verify that $V_{n}$ is open suppose $x_{0} \in V_{n}$; then $x_{0} \in V$ so by construction $V_{n}$ contains an interval along each $R_{x}^{y}$, as $V$ does, and $V(x)=V_{n}(x)$, 
so $x \in \operatorname{int} V_{n}(x)$.

The space $S(X)$ is never metrizable. However an appropriate uniform version of it is metrizable, as will be noted in $\S 4$, when $X$ is first-countable.

The most interesting property of $S(X)$ is perhaps the fact that the decomposition $\left\{S_{x}: x \in X\right\}$ can be recovered from the space $S(X)$; in fact the fibers $S_{x}$ are the path components of $S(X)$. This is in sharp contrast to most results expressing one class of spaces as the quotients of another class; typically the decomposition must be given along with the representing space. Another interesting property is that $S(X)$ has essentially the same sort of connectedness as $X$. Again most results involving representation as quotients lead to disconnected representing spaces.

2.5. Suppose $f: Y \rightarrow S(X)$ is a map, and $Y$ is connected and locally connected. Then $f[Y]$ lies entirely in some $S_{x}$.

Proof. Any point of $S_{x}$ has a neighborhood for which the component containing the point lies entirely in $S_{x}$. Since $Y$ is locally connected each $f^{\leftarrow}\left[S_{x}\right]$ is open, and thus each $f^{\leftarrow}\left[S_{x}\right]$ is closed; so $f[Y]$ lies entirely in some $S_{x}$.

2.6 (COROLlaRY). The path components of $S(X)$ are the fibers $S_{x}$ of $\pi$. That is, $X$ is the path component space of $S(X)$.

2.7. The connected subsets of $X$ are precisely the images of the connected subsets of $S(X)$. In particular the components of $S(X)$ are the preimages of the components of $X$.

Proof. The map $\pi$ is hereditarily quotient, so each restriction of it to a subspace of $X$ is a quotient map with connected fibers. Thus a set is connected if and only if its preimage in $S(X)$ is connected, from which the results follow immediately.

In studying further the properties of $S(X)$ a particular type of neighborhood is important; the neighborhood $V$ of $x_{0}$ is simple if there is an open set $W$ with $x \in W$ such that $V_{a}^{b}$ is initial precisely when $x=a$ and $b \in X, V_{a}^{b}$ is terminal precisely when $a \in W$ and $b=x, V_{a}^{b}=\varnothing$ in all other cases, and $V_{0}=\left\{x_{0}\right\}$. Clearly in this case $\pi[V]=W$, and $V$ is said to be built on $W$.

2.8. (a) Every uniform neighborhood is simple. 
(b) The simple neighborhoods of $x_{0}$ form a base at $x_{0}$.

(c) The image of any base at $x_{0}$ is a base at $x$.

The following characterization of the closure operator in $S(X)$ is a very useful formulation. Given $B \subset S(X)$ set $B^{+}(x)=\left\{y: B_{x}^{y}\right.$ is cofinal $\}$ for each $x, B^{0}=\left\{x_{0}: x \in \operatorname{cl} B^{+}(x)\right\}$, and $B^{*}=\bigcup_{x, y \in X} \operatorname{cl} B_{x}^{y}$. It is clear that $B^{*}$ is the closure of $B$ in the disjoint sum topology of the $S_{x}$.

\section{9. $\operatorname{cl} B=B^{*} \cup B^{0}$.}

Proof. Certainly $B^{*} \subset \mathrm{cl} B$. If $x_{0} \in B^{0}$ and $V$ is a neighborhood of $x$, then there is $y \in V$ such that $B_{x}^{y}$ is cofinal, so any neighborhood of $x_{0}$ built on $V$ meets $B$. Thus $B^{\circ} \subset \mathrm{cl} B$, so $B^{*} \cup B^{0} \subset \mathrm{cl} B$.

If $p \notin B^{*}$ is radial then clearly $p \notin \mathrm{cl} B$. If $x_{0} \notin B^{*}$ then no $B_{x}^{y}$ is initial, so there is an interval from $x_{0}$ along each ray $R_{x}^{y}$ that is disjoint from $B$. Similarly if $x_{0} \notin B^{0}$ there is a neighborhood $V$ of $x$ such that no $B_{y}^{x}$ is cofinal with $y \in V$; that is, there are terminal sets which do not meet $B$ along each $R_{y}^{x}$ with $y \in V$. It follows that if $x_{0} \notin B^{*} \cup B^{0}$ then $x \notin \mathrm{cl} B$.

A very important property of the spaces $S(X)$ is that they may be characterized by their Stone-Čech compactifications.

2.10. $S(X)$ and $S(Y)$ are homeomorphic if and only if $\beta S(X)$ and $\beta S(Y)$ are homeomorphic.

Proof. It is clear that each point of $S(X)$ is the limit of a sequence of distinct points, and by 2.4 every point of $S(X)$ is a $G_{\hat{o}}$. The stated result is now immediate from $[3,9 \mathrm{~N}]$.

REMARK. It is clear that the given construction is very special and can be greatly generalized. Needed for the general construction is a space $R_{x}^{y}$ and two open filters $\sigma_{x}^{y}$ and $\tau_{x}^{y}$ for each pair of points $x, y$. The members of $\sigma_{x}^{y}$ take the place of initial sets on $R_{x}^{y}$ and the members of $\tau_{x}^{y}$ take the place of terminal sets. Then the properties of $S(X)$ so constructed will depend primarily upon the properties of the two point extensions of $R_{x}^{y}$ obtained by adjoining points have trace filters $\sigma_{x}^{y}$ and $\tau_{x}^{y}$.

Another generalization begins from $S(X)$ as a weaker topology on the product $D \times S$, where $D$ is the discrete space on the set $X$ and $S$ is the quotient space $(D \times R \cup\{0\}) / D \times\{0\}$. 
3. The function $S(f)$. Given any function $f: X \rightarrow Y$ define $S(f): S(X) \rightarrow S(Y)$ by $S(f)\left(x_{0}\right)=f(x)_{0}$ for $x_{0} \in X_{0}$ and $S(f)(p)=p \in R_{f(x)}^{f(y)}$ when $p \in R_{x}^{y}$.

The function $S(f)$ has properties closely related to those of $f$. Some of these are given below, where we write $\mathscr{P} \triangle \mathscr{Q}$ to indicate that $S(f)$ has property $\mathscr{P}$ if and only if $f$ has property $\mathscr{Q}$. Also note that quotient, pseudo-open, and biquotient functions are always considered to be onto.
3.1. (a) one-to-one $\triangle$ one-to-one
(b) onto $\triangle$ onto
(c) $\operatorname{map} \triangle \operatorname{map}$
(d) homeomorphism $\triangle$ homeomorphism
(e) embedding $\triangle$ embedding
(f) quotient $\triangle$ hereditarily quotient (=pseudo-open)
(g) pseudo-open $\triangle$ pseudo-open
(h) biquotient $\triangle$ biquotient
(i) closed $\triangle$ closed and finite-to-one
(j) open $\triangle$ open and onto
(k) closed embedding $\triangle$ closed embedding
(l) open embedding $\triangle$ homeomorphism onto.

Proof. For convenience write $g=S(f)$.

(a) and (b) are straightforward, (d) follows from (a), (b), and (c), (k) follows from (e) and (i), and (l) follows from (e) and (j).

(c) That $g$ is a map when $f$ is follows from the equality $g^{-}[V(f(x))]=W(x)$. The converse follows from the facts that $\pi_{X}$ is quotient and $\pi_{Y}$ is a map.

(e) Suppose $f$ is an embedding. In particular it is one-to-one, so $g$ is an embedding on radial points. Given a simple neighborhood $V$ of $x_{0}$, based on $W$, choose an open $U \subset Y$ so that $W=f^{\leftarrow}[U]$, and set $T=\cup\left\{R_{u}^{v}: u \in U-f[X], v \in Y\right\} \cup \cup\left\{R_{f(x)}^{v}: v \notin f[X]\right\}$. Then $T$ is open, $T \cap Y_{0}=\varnothing$, and $g^{\leftarrow}[T]=\varnothing$. Setting $Z=T \cup g[V]$, and noting that $V=g^{\leftarrow}[g[V]]$, it follows that $V=g^{\leftarrow}[Z]$. The set $Z$ is certainly open at each radial point, and contains the single central point $y_{0}=f(x)_{0}$. The set $g[V]$ is initial on each $R_{y}^{v}$, for $v \in f[X]$, and the set $T$ is initial on the remaining $R_{y}^{v}$. The set $g[V]$ is terminal at least for all $R_{u}^{y}$ with $u \in U \cap f[X]$, and the set $T$ is terminal for the remaining $u \in U$. Thus $Z$ is an open set.

Suppose $g$ is an embedding and $V$ is an open set in $X$. For each $x \in V$ choose a simple neighborhood $H_{x}$ of $f(x)_{0}$, based on $W_{x} \subset Y$, such that $g^{-}\left[H_{x}\right] \subset V_{x}(2.1)$. Then $V \subset \cup f^{\leftarrow}\left[W_{x}\right]$. Setting $U=\cup W_{x}$, it follows that $V \subset f^{\leftarrow}[U]$. Now if $f(z) \in U$ then $f(z) \in W_{x}$ for some 
$x \in V$. If $f(z)=f(x)$ then $z=y \in V$. If $f(z) \neq f(x)$ then $f(z)=\pi(p)$, where $p \in R_{c}^{d} \cap W_{x}$ with $0=f(z) \neq f(x)$ and thus $d=f(x)$. But this gives $p \in g(q)$ where $q \in g^{-}\left[W_{x}\right] \subset V_{x}(2.1)$, so $z=\pi(q) \in V$. Thus $f^{\leftarrow}[U] \subset V$. It follows that $f$ is an embedding.

(f) and (g). Suppose $g$ is quotient, and $f^{\leftarrow}(y) \subset U$, with $U$ open. Set $T=\left\{w_{0}: w \in f^{\leftarrow}(y)\right\} \cup\left\{R_{u}^{v}: v \in X, u \in f^{\leftarrow}[f[U]]\right\}$. Then $T$ is open and $T=g[g[T]]$, so $g[T]$ is open, and $y_{0} \in g[T]$, which implies $y \in$ int $T(y) \subset f[U]$. Thus if $g$ is quotient then $f$ is pseudo-open.

Suppose $f$ is pseudo-open, and $g^{\leftarrow}\left(y_{0}\right) \subset U$, where $U$ is open. Set $V=U\left\{\right.$ int $\left.U(x): x_{0} \in g^{\leftarrow}\left(y_{0}\right)\right\}$. Then $V$ is open, and $f^{\leftarrow}(y) \subset V$, so $y \in$ int $f[V]$. Certainly $y_{0} \in g[U] ; g[U]$ is initial on every $R_{y}^{f(z)}, z \in X$, and terminal on every $R_{f(z)}^{y}, z \in U(x)$, in particular on int $f[V]$. It follows that $y_{0} \in \operatorname{int} g[V]$.

The argument when $g(p) \subset U$ and $p$ is radial is trivial.

(h) The argument is similar to that for (f) and (g).

(i) If $f$ is not finite-to-one, choose $y \in Y$ and a sequence $\left\{x_{n}\right\}_{n=1}^{\infty}$ of distinct members of $f^{\prime}(y)$. Let $x$ be any point of $X$, and set $B=\left\{p_{n}\right\}_{n=1}^{\infty}$ where $p_{n}$ is the point $1+1 / n$ on the ray $R_{x}^{x_{n}}$. Then $B$ is closed in $S(X)$ and $g[B]$ is not closed in $S(Y)$.

It follows from the facts that $\pi_{X}$ is a map and $\pi_{1}$. is a quotient that $f$ must be closed when $g$ is closed.

Now suppose $f$ is closed and finite-to-one, and $B$ is closed in $S(X)$. Set $C=g[B]$. Since each fiber is finite, it follows that $C_{a}^{b}$ is initial (terminal) if and only if some $B_{c}^{d}$ is initial (terminal) where $f(c)=a$ and $f(d)=b$. In particular $C^{+}(y)=\cup\left\{f\left[B^{+}(x): x \in\right.\right.$ $\left.\left.f^{\leftarrow}(y)\right]\right\}$, and the union is finite. Since $f$ is a closed map, cl $f\left[B^{+}(x)\right]=$

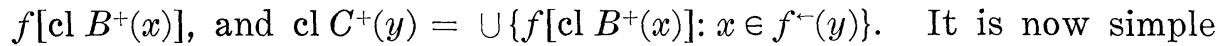
to conclude from 2.9 that $C$ is closed.

(j) If $S(f)$ is open, $U \subset X$ is open, and $y \in f[U]$, then $y=f(x)$, where $x_{0} \in \pi^{\leftarrow}[U]$. This gives $y_{0} \in g\left[\pi^{\leftarrow} f[U]\right]$, and so $y \in \operatorname{int} f[U]$. Thus $f$ is open. To see that $f$ is onto, note that if $y \notin f[X]$ then $g[S(X)]$ is not a neighborhood of any of its central points, so $g$ is not open.

If $f$ is open and onto, $U \subset S(X)$ is open, and $y_{0}=g\left(x_{0}\right)$ is a central point of $g[U]$, there is $V$ open in $X$ with $x \in V$ and $V \in U(x)$. Then $f[V]$ is open in $Y, y \in V, g[U]$ is terminal on every $R_{f(z)}^{y}$ for which $z \in V$, and $g[U]$ is initial on every $R_{y}^{f(z)}$ with $z \in X$; thus $g[U]$ is a neighborhood of each of its central points. Clearly it is also a neighborhood of each of its radial points, so $g[U]$ is open.

4. Construction of $U(X)$. There is a more uniform version of $S(X)$ that is useful in connection with metrizability questions and related topics. Given any open subset $V$ of $X$, any point $x \in V$, and any integer $n$, define 


$$
U_{n}(x)=\left\{x_{0}\right\} \cup\left\{(0,1 / n)_{x}^{y}: y \in X\right\} \cup\left\{(k-1 / n, k+1 / n)_{y}^{x}: y \in V, k>n\right\} .
$$

The space $U(X)$ has the same ground set as $S(X)$, neighborhoods of central points $x_{0}$ are sets $V_{n}(x)$, where $V \subset X$ is open and $x \in V$, and neighborhoods of radial points are the same as in $S(X)$.

The local character at a point of a space is the minimum cardinal of a base at the point; the local character of a space is the supremum of the local characters of its points. The metricity (see [4, pg. 5]) is the least cardinal of a family, consisting of discrete collections of open sets, whose union is a basis for the space.

4.1. (a) The local character at $x_{0} \in U(X)$ is the product of $\omega_{0}$ with the local character at $x \in X$.

(b) The local character of the space $U(X)$ is the product of $\omega_{0}$ with the local character of the space $X$.

(c) The metricity of the space $U(X)$ is the product of $\omega_{0}$ with the local character of the space $X$.

Proof. The product with $\omega_{0}$ is necessary in the above results only when $X$ has a point with finite local character; finite local character can only be equal to 1 , of course.

(a) and (b) are easily established using 2.8.

To show (c), note first that the local character of a space is never larger than its metricity, thus by (b) the metricity of $U(X)$ is not larger than the product of $\omega_{0}$ with the local character of $X$. To see the converse, note that if $\left\{W^{X}\right\}$ is any family of open sets in $X$ with $x \in W^{X}$ for each $x \in X$, the family $\left\{W_{n}^{X}(x)\right\}$ is a discrete family of open sets in $U(X)$. If the cardinal $\omega_{\alpha}$ is the local character of $X$ then there are $\omega_{\alpha}$ families of open sets in $X$, each family indexed by the points of $X$, such that the union of the families is a base for $X$. The union of the corresponding discrete families in $U(X)$ forms a base for central points in $U(X)$. Using the fact that $R$ has an $\omega_{0}$-discrete base it is easily seen that the metricity of $U(X)$ is not greater then the product of $\omega_{0}$ with the local character of $X$.

A variety of properties of $U(X)$ are equivalent to the firstcountability of $X$. The following lemma is used to establish some of them.

4.2. Let $x$ be a point of $X$ that does not have a countable local base. Then if $\left\{V_{n}\right\}_{n=1}^{\infty}$ is any countable family of neighborhoods of $x_{0} \in U(X)$ there is a free z-ultrafilter $M^{p}$ on $U(X)$ such that $p \in$ $\mathrm{cl}_{\beta U(X)} V_{n}$ for each $n$. 
Proof. It is clear that $\left\{V_{n}\right\}$ can be assumed to be a decreasing family of simple neighborhoods built on the family $\left\{W_{n}\right\}$ of open neighborhoods of $x$. There must be an open neighborhood $T$ of $x$ and a point $y_{n} \in W_{n}-T$ for each $n$; also it can be assumed that $\left\{y_{n}\right\}$ is a sequence of distinct points. For each $n$ there is $p_{n} \in V_{n} \cap$ $R y_{n}^{r}$; setting $P=\left\{p_{n}\right\}$ and noting that $x_{0} \in \pi-[T] \subset U(X)-P$ it follows that $P$ is a closed discrete infinite subset of $U(X)$.

Recall that a space $Y$ is a (strict) $p$-space if in $\beta Y$ there is a sequence $\left\{\gamma_{n}\right\}_{n=1}^{\infty}$ of collections of open sets such that for each $y \in Y$, $y \in \bigcap_{n=1}^{\infty} \operatorname{St}\left(y, \gamma_{n}\right) \subset Y$ (and for each $\left.n \operatorname{cl}_{\beta Y} \operatorname{St}\left(y, \gamma_{n+1}\right) \subset \operatorname{St}\left(y, \gamma_{n}\right)\right)$.

4.3. The following are equivalent for any space $X$.

(a) The space $U(X)$ is a strict p-space.

(b) The space $U(X)$ is a p-space.

(c) Every point of $U(X)$ is a $G_{o}$ in $\beta U(X)$.

(d) Every point of $U(X)$ has countable character in $\beta U(X)$.

(e) $U(X)$ is metrizable.

(f) $U(X)$ is first-countable.

(g) $X$ is first-countable.

Proof. According to 4.1 conditions (e), (f), and (g) are equivalent. According to [3, 9.7] (f) and (d) are equivalent. Trivially (a) implies (b) and (d) implies (c). Since any metrizable space is a strict $p$-space, (e) implies (a). Thus it need only be shown that (b) implies (g) and that (c) implies (g). These two implications follow easily from 4.2 .

The following result is established in exactly the same way as 2.10 .

4.4. The space $U(X)$ and $U(Y)$ are homeomorphic if and only if the spaces $\beta U(X)$ and $\beta U(Y)$ are homeomorphic.

5. Categorical remarks. This section consists of remarks pointing out various categorical embeddings implicit in the previous constructions.

Let $P(X)$ be the quotient space obtained from a space $X$ through identifying points in the same path component. Noting that every map between spaces respects this equivalence relation, it follows that $P$ is a functor from Top to Top (see [5], where it is treated as a functor from Top to Set).

According to $3.1 S$ is a functor from Top to Top, and according to 2.2 and $2.6 P(S(X))=X$ for each space $X$. Clearly $P(S(f))=f$ 
for each map $f$, and it follows that $P S$ is the identity functor from Top to Top. Also it is clear that these remarks hold for $U$ as well.

5.1. The functors $S$ and $U$ are embeddings from the category of topological spaces and maps into the category of paracompact Hausdorff spaces and maps.

The following concequence of 4.3 is worth separate statement.

5.2. The functor $U$ is an embedding of the category of firstcountable topological spaces and maps into the category of metrizable spaces and maps.

The results 2.10 and 4.4 lead immediately to the following:

5.3. The functors $\beta S$ and $\beta U$ are embeddings of the category of topological spaces and maps into the category of compact Hausdorff spaces. The interest in the preceding remarks is not in the category theoretic fact that the embeddings exist, but in the ease with which the topological features of the embeddings can be ascertained.

\section{REFERENCES}

1. A. V. Arhangel'skii, Some types of factor mappings and the relations between classes of topological spaces, Soviet Math. Dokl., 4 (1963), 1726-1729.

2. C. J. R. Borges, On stratifiable spaces, Pacific J. Math., 17 (1966), 1-16.

3. L. Gillman and M. Jerison, Rings of continuous functions, Van Nostrand, New York, 1960.

4. M. E. Rudin, Lectures on Set Theoretic Topology, Regional Conference Series in Mathematics 23, American Mathematical Society, Providence, 1975.

5. E. H. Spanier, Algebraic Topology, McGraw-Hill, New York, 1966.

Received April 19, 1976 and in revised form December 18, 1977.

Marquette University

Milwaukee, WI 53233 


\section{PACIFIC JOURNAL OF MATHEMATICS}

\section{EDITORS}

DoNALD BABBITT (Managing Editor)

University of Galifornia

Los Angeles, California 90024

HUgo RossI

University of Utah

Salt Lake City, UT 84112

C. C. MOORE AND ANDREW OGG

University of California

Berkeley, CA 94720
J. DugunduI

Department of Mathematics

University of Southern California

Los Angeles, California 90007

R. Finn and J. Milgram

Stanford University

Stanford, California 94305

\section{ASSOCIATE EDITORS}
E. F. BeCKenbaCh
B. H. NeumanN
F. WOLF
K. YoSHIDA

\section{SUPPORTING INSTITUTIONS}

UNIVERSITY OF BRITISH COLUMBIA

CALIFORNIA INSTITUTE OF TECHNOLOGY

UNIVERSITY OF CALIFORNIA

MONTANA STATE UNIVERSITY

UNIVERSITY OF NEVADA, RENO

NEW MEXICO STATE UNIVERSITY

OREGON STATE UNIVERSITY

UNIVERSITY OF OREGON
UNIVERSITY OF SOUTHERN CALIFONIA STANFORD UNIVERSITY UNIVERSITY OF HAWAII UNIVERSITY OF TOKYO UNIVERSITY OF UTAH WASHINGTON STATE UNIVERSITY UNIVERSITY OF WASHINGTON 


\section{Pacific Journal of Mathematics}

\section{Vol. 91, No. $1 \quad$ November, 1980}

Harvey Leslie Abbott, Extremal problems on nonaveraging and nondividing

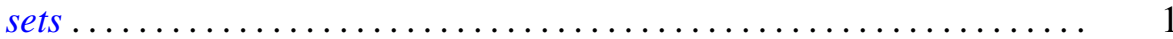

Marine Bruce Abrahamse and Stephen D. Fisher, Mapping intervals to

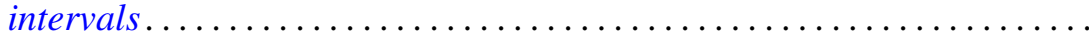

William Wells Adams, The best two-dimensional Diophantine

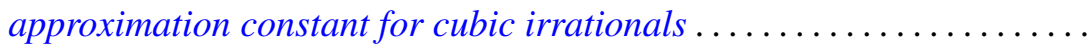

Marilyn Breen, A quantitative version of Krasnosel'skiu 's theorem in

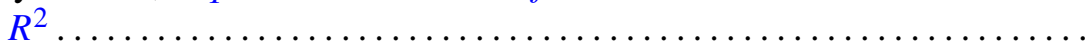

Stephen LaVern Campbell, Linear operators for which $T^{*} T$ and $T T^{*}$

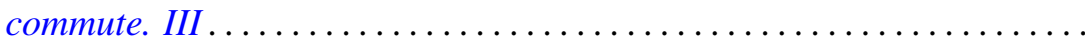

Zvonko Cerin, On cellular decompositions of Hilbert cube manifolds ......

J. R. Choike, Ignacy I. Kotlarski and V. M. Smith, On a characterization

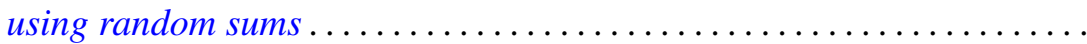

Karl-Theodor Eisele, Direct factorizations of measures .............. 79

Douglas Harris, Every space is a path component space ............. 95

John P. Holmes and Arthur Argyle Sagle, Analytic H-spaces, Campbell-Hausdorff formula, and alternative algebras.............

Richard Howard Hudson and Kenneth S. Williams, Some new residuacity criteria ..........................................

V. Karunakaran and Michael Robert Ziegler, The radius of starlikeness for a class of regular functions defined by an integral ....

Ka-Sing Lau, On the Banach spaces of functions with bounded upper

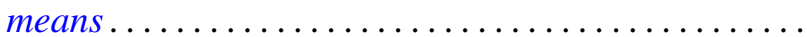

Daniel Paul Maki, On determining regular behavior from the recurrence formula for orthogonal polynomials................

Stephen Joseph McAdam, Asymptotic prime divisors and going down...

Douglas Edward Miller, Borel selectors for separated quotients ..

Kent Morrison, The scheme of finite-dimensional representations of an algebra

Donald P. Story, A characterization of the local Radon-Nikodým property by tensor products

Arne Stray, Two applications of the Schur-Nevanlinna algorithm ...

N. B. Tinberg, The Levi decomposition of a split $(B, N)$-pair ...

Charles Irvin Vinsonhaler and William Jennings Wickless, A theorem on quasi-pure-projective torsion free abelian groups of finite rank... 